Disponível em

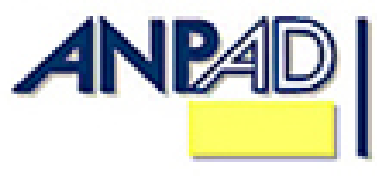

http://www.anpad.org.br/rac

RAC, Rio de Janeiro, v. 16, n. 2, art. 5, pp. 253-270, Mar./Abr. 2012

$(\infty)$ EY-No

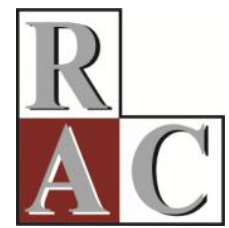

\title{
Bem-Estar no Trabalho: um Estudo sobre suas Relações com Clima Social, Coping e Variáveis Demográficas
}

\author{
Work Well-Being: a Study of Its Relations with Social Climate, Coping and \\ Demographics Variables
}

Fábio Rocha Sobrinho * E-mail: fabiorochasobrinho@gmail.com Universidade Católica de Brasília - UCB Brasília, DF, Brasil.

Juliana Barreiros Porto

E-mail: jbporto@unb.br Universidade de Brasília - UnB Brasília, DF, Brasil.

\footnotetext{
* Endereço: Fábio Rocha Sobrinho

Universidade Católica de Brasília - UCB, Campus Avançado Asa Norte, SGAN 916, Avenida W5, Brasília/DF, 70790-160.
}

Copyright (C) 2012 RAC. Todos os direitos, até mesmo de tradução, são reservados. É permitido citar parte de artigos sem autorização prévia, desde que seja identificada a fonte. 


\title{
Resumo
}

O presente estudo teve como objetivo identificar o impacto de variáveis demográficas, do clima social e coping no bem-estar no trabalho. Para abordar bem-estar foi utilizado modelo que unifica as correntes eudaimônicas e hedonistas, e foram utilizadas as escalas de bem-estar no trabalho, compostas pelos fatores: realização no trabalho, afetos positivos e negativos; clima social. Esta, por sua vez, composta pelos aspectos: inovação e desempenho, reconhecimento e autonomia, adaptada para o contexto organizacional, validada no estudo e coping, composta pelos fatores: esquiva, manejo e controle. Participaram da pesquisa 2.438 funcionários de uma organização confessional do ramo de educação, que corresponde a 58\% da população. As análises de correlação e regressão demonstraram que os fatores de clima impactam no bem-estar, dentre esses, autonomia apresentou correlação positiva e de moderada a alta com bem-estar, e os fatores inovação, desempenho e reconhecimento apresentaram correlações mais fortes. Nos fatores de coping, esquiva teve resultado fraco na correlação, porém, manejo e controle tiveram resultado significativo. Concluiu-se que o clima social e as estratégias de enfrentamento do estresse impactam no bem-estar no trabalho. Apesar das contribuições, foram limitações a coleta ter sido realizada pela própria organização, e o estudo se restringir a uma organização apenas.

Palavras-chave: clima social; clima organizacional; felicidade; coping; bem-estar no trabalho.

\begin{abstract}
The current study has as its main goal to identify the influence demographic variables, social climate and coping have on work well-being. Towards this end, we used scales of work well-being composed by three factors: selffulfillment, negative and positive affects, and social climate, all adapted to the organizational context and composed by the factors of innovation, performance, recognition, autonomy, and coping as composed by control, escape and management. A sample of 2,483 employees from a Religious educational organization took part in this research, which corresponds to $58 \%$ of the organization's population. As observed, age and education levels positively influence the variation of work well-being. Correlation and regression analysis show that all social climate factors influence work well-being; in particular, autonomy presented a moderate to high positive correlation, and recognition with work well-being and the factors innovation and performance showed the strongest correlations. Factors of coping-appraisal focus and emotion focus had stronger impact on the results. The conclusion is that social climate and mastering coping have meaningful roles for predicting work wellbeing. Limitations of this paper are the fact that data were collected by the organization and the study was done in only one organization.
\end{abstract}

Key words: social climate; organizational climate; happiness; coping; work well-being. 


\section{Introdução}

O estudo da felicidade tem crescido nos últimos anos. Segundo Ryan e Deci (2001), o estudo desse tema está focado na busca da compreensão das experiências de prazer versus desprazer. De acordo com Tamayo (2004), a felicidade é uma preocupação científica. E como afirmam Siqueira e Padovam (2008), os pesquisadores têm estudado esse tema complexo e buscado identificar o quanto as pessoas se consideram felizes.

Para Albuquerque e Tróccoli (2004), com o passar dos anos a literatura da área adotou o bemestar como um sinônimo de felicidade. Segundo Paschoal (2008), diversos autores têm se dedicado a desenvolver pesquisas para compreender o bem-estar, principalmente pelo fato de entenderem que este corresponde a mais de uma esfera da vida, como o trabalho, por exemplo.

Para Tamayo (2004), o tempo dedicado ao trabalho, que representa uma parte significativa da vida do indivíduo, consiste num componente fundamental para a construção e o desenvolvimento do bem-estar pessoal e da felicidade. Assim, é fundamental o estudo do bem-estar no trabalho.

Apesar de escassos, nas últimas décadas surgiram estudos empíricos com o objetivo de entender as variáveis envolvidas na predição do bem-estar no trabalho. Autores como Walton (1973), Werther e Davis (1983), Nadler e Lawler (1983), Diener (1984), Brief e Roberson (1989), Ryff (1989), Waterman (1993), Warr (1994, 2006), Weiss e Cropanzano (1996), Daniels (2000), Horn, Taris, Schaufel e Schreurs (2004), Paz (2004), Dessen e Paz (2010a), Paschoal (2008), Paschoal, Torres e Porto (2010), entre outros, ressaltaram a importância de se estudar o bem-estar relacionado ao trabalho, e desenvolveram modelos teóricos e empíricos sobre a estrutura desse fenômeno e as variáveis situacionais e pessoais que o determinam.

Este estudo busca contribuir com a literatura do bem-estar no trabalho, a partir da identificação de variáveis que se relacionam a este fenômeno. Especificamente, tem o objetivo de identificar o impacto de variáveis demográficas, clima social e coping no bem-estar no trabalho. Assim, será apresentado um breve referencial sobre as variáveis do estudo, para, em seguida, apresentar o método, os resultados e a discussão da pesquisa empírica.

\section{Bem-Estar}

A concepção de bem-estar adotada neste estudo está fundamentada na psicologia positiva que o considera como sinônimo de felicidade, enfatizando os aspectos positivos da experiência do trabalhador. Assim, aspectos como estresse ou esgotamento no trabalho que caracterizam aspectos negativos deste são correlacionados a bem-estar, mas não caracterizam bem-estar no trabalho propriamente dito (Paschoal, Torres, \& Porto, 2010).

O número de pesquisas na área de bem-estar está crescendo, segundo Albuquerque e Tróccoli (2004), e sobre o tema derivam duas perspectivas gerais: (a) hedônica, cujo foco está na felicidade e define bem-estar subjetivo nos termos das ligações de prazer e desprazer; e (b) eudaimônica, cujo foco está no significado da auto-realização e define bem-estar psicológico em termos do grau de satisfação que uma pessoa tem em sua vida em geral. De acordo com esses autores, a principal diferença entre elas reside na concepção de felicidade adotada. De acordo com Ryan e Deci (2001), os debates entre os teóricos hedônicos e eudaimônicos são frequentemente intensos. Porém, há uma preocupação em aproximar estas duas posições, pela importância prática e teórica de ambos os conceitos.

Bem-estar subjetivo tem sido concebido como índice primário do bem-estar desde as décadas passadas, e muitas das pesquisas da área revisaram os estudos que empregam o bem-estar subjetivo 
como a principal variável adquirida, principalmente ao discutir os princípios da chamada felicidade. Mas, qual a relação entre a felicidade eudaimônica e a felicidade hedônica?

Para Waterman (1993), o bem-estar é um estado afetivo, de modo que os afetos positivos prevalecem sobre os negativos, e tanto a expressão pessoal quanto a auto-realização referem-se à experiência subjetiva de desenvolvimento de habilidades, realização dos próprios potenciais e avanços de seus propósitos na vida.

Portanto, evidências encontradas nessas diferentes pesquisas indicam que o bem-estar, provavelmente, é melhor conceituado como fenômeno multidimensional, que inclui aspectos de ambas as concepções, hedônicas e eudaimônicas, conceito este adotado pelo presente estudo.

\section{Bem-estar no trabalho}

A revisão de pesquisas sobre bem-estar ocupacional evidencia que esse campo de estudos tem sido marcado por algumas confusões conceituais, com a adoção de termos como qualidade de vida, satisfação e afeto no trabalho. Algumas vezes, as definições destes termos se aproximam na conceituação e, em outros estudos, distanciam-se. Esta falta de clareza também se apresenta nos indicadores desses construtos, em diferentes dimensões, física, psicológica e social.

Do ponto de vista sócio-técnico, a qualidade de vida no trabalho deriva da satisfação das necessidades básicas dos empregados. De acordo com Brooks e Anderson (2005), essa perspectiva teve origem nos estudos realizados no Instituto Tavistock, de Londres, em 1950, por Trist et al., cujo objetivo era promover a reorganização do trabalho mediante a análise e a reestruturação da tarefa, como forma de otimizar as metas organizacionais de produtividade e as necessidades dos empregados.

Fundamentados nesta perspectiva, de acordo com Rodrigues (1995), os anos seguintes foram marcados pelo surgimento de vários modelos (House, 1981; Nadler \& Lawler, 1983; Walton, 1973; Werther \& Davis, 1983). Esses modelos foram desenvolvidos com a preocupação básica de caracterizar e especificar as diferentes dimensões da tarefa e da organização, associadas à qualidade de vida no trabalho. Além disso, objetivavam fornecer subsídios ao desenvolvimento de programas voltados à implementação de mudanças organizacionais destinadas a aumentar a satisfação do trabalhador. O movimento de qualidade de vida no trabalho estimulou não apenas a realização de pesquisas sobre seus determinantes, mas também sobre suas consequências, ou seja, sobre os indicadores individuais de bem-estar e saúde no trabalho.

Por muito tempo, o afeto no trabalho foi considerado como a satisfação no trabalho, a principal manifestação de bem-estar ali (Sousa-Poza \& Sousa-Poza, 2000). Todavia, de acordo com Brief e Roberson (1989), as últimas décadas foram marcadas por descobertas que demonstraram que o bemestar ocupacional não envolve apenas elementos afetivos como a satisfação no trabalho.

Nessa perspectiva, Horn et al. (2004) basearam-se na teoria de Ryff (1989) ao explicarem que, apesar do afeto ser uma dimensão central do conceito de bem-estar ocupacional, ele pode ser entendido como a avaliação positiva de várias dimensões do trabalho, especificamente afetiva, profissional, social, cognitiva e psicossomática. Além disso, diferentes estudos apontam as características do ambiente de trabalho como um importante determinante do bem-estar do empregado, desde que facilitem a aquisição de metas pessoais (Doest, Maes, Gebhardt, \& Koelewijn, 2006). Contudo, de acordo com Warr (2006), existem três outros fatores que devem ser considerados na concepção do bem-estar ocupacional: (a) o processo cognitivo usado para interpretar a situação; (b) opiniões de outras pessoas; e, (c) traços de personalidade.

Segundo Horn et al. (2004), o bem-estar não se reduz à avaliação afetiva dos fatos da vida, é mais que isto, cobre dimensões como estado emocional, motivação, competência, eficácia do indivíduo. Assim, a definição de bem-estar ocupacional de Horn et al. (2004) está calcada na perspectiva do bem-estar psicológico. 
De acordo com Paschoal (2008), por sua vez, o termo afeto no trabalho surge em diferentes estudos, sustentado na literatura de humores e emoções. Nesses estudos também foi constatado que a experiência afetiva é mais do que a satisfação no trabalho. As emoções e humores vivenciados no trabalho poderiam ser considerados as causas da satisfação no trabalho ou de indicadores dela (Brief \& Weiss, 2002). Essa consideração corrobora os estudos de Weiss e Cropanzano (1996), ao definirem a satisfação como um julgamento avaliativo acerca do trabalho. De acordo com Diener (1984), a satisfação com a vida e os afetos positivos e negativos são sub-dimensões do bem-estar subjetivo. Corroborando esta afirmação, Waterman (1993) demonstra que tanto as emoções prazerosas quanto a percepção de realização são importantes. Assim, situações diferentes levam a experiências de bemestar distintas. Já de acordo com Waterman (1993), o bem-estar pode ser considerado como o estado afetivo do indivíduo e como a percepção de expressão e desenvolvimento de potenciais e avanço dos propósitos de vida. Dessa forma, são considerados critérios das perspectivas hedônica e eudaimônica.

Ainda sobre o assunto, Daniels (2000) afirma que o bem-estar ocupacional pode ser considerado o bem-estar afetivo no trabalho, a prevalência das emoções positivas. De acordo com Paschoal (2008), definindo bem-estar ocupacional desta forma, Daniels (2000) dá a merecida relevância às respostas do indivíduo, às experiências resultantes das interações do ambiente de trabalho e às suas características pessoais.

Paz (2004) pressupõe que as características pessoais mediam o impacto da organização sobre os indivíduos que nela trabalham, afetando assim o bem-estar destes seres, a depender da subjetividade de cada um. Neste sentido, Paz (2004) define bem-estar pessoal nas organizações como a satisfação de necessidades e realização de desejos dos indivíduos no desempenho de seu papel organizacional, e apresenta dois polos, os quais o indivíduo pode experimentar: a gratificação e o desgosto. De acordo com Paz (2004), a gratificação seriam valorização do trabalho, reconhecimento pessoal, autonomia, expectativa de crescimento, suporte ambiental, recursos financeiros e orgulho. O descontentamento seria o sentimento de mal-estar no indivíduo mediado por diferentes situações (desvalorização do seu trabalho, medo de não atender às exigências da organização, falta de habilidade para conseguir imprimir o seu estilo pessoal na execução de suas tarefas, falta de condições adequadas de trabalho para o alcance do desempenho esperado, percepção de injustiça salarial, e sentimento de frustração por pertencer à organização). Apesar de o desgosto estar presente na definição, sendo avaliado pela inversão do polo oposto, são enfatizados os indicadores de gratificação, que contribuem para que as empresas se mantenham no mercado com produtividade e bem-estar dos funcionários (Paz, 2004).

É possível constatar até o momento em que o afeto consiste num elemento essencial do bemestar ocupacional, assim como defende Daniels (2000), porém não se pode ignorar a percepção de expressão e auto-realização, que propõe Waterman (1993) e, ainda, a proposta semelhante de Paz (2004) sobre a realização de desejos dos indivíduos. Considerando-se que todas as abordagens versam sobre a problemática da saúde no trabalho, sendo esta tratada como o bem-estar físico, mental e social do trabalhador, e não simplesmente como a ausência de doença (Paz, 2005), torna-se fundamental o estudo do bem-estar pessoal também no contexto organizacional.

Uma vez que esse estudo considera a perspectiva hedonista e eudaimônica do bem-estar e, ainda, considerando que o bem-estar ocupacional engloba tanto aspectos afetivos quanto cognitivos, o conceito de bem-estar ocupacional adotado neste estudo defende a definição de Paschoal (2008, p. 23). A autora define o bem-estar ocupacional como a "prevalência de emoções positivas no trabalho e a percepção do indivíduo de que, no seu trabalho, expressa e desenvolve seus potenciais/habilidades e avança no alcance de suas metas de vida." 


\section{Clima Social}

Puente-Palacios (2002) afirma que a cultura organizacional é composta por um conjunto de elementos como normas, valores, crenças, regras, e constitui um modelo de definição de padrões de comportamento construídos ao longo da história da organização, capaz de influenciar o comportamento dos seus membros. Portanto, clima organizacional compreende um conjunto de percepções globais compartilhadas pelos indivíduos, referente às variáveis organizacionais que compõem o ambiente de trabalho.

Segundo Puente-Palacios e Carneiro (2005), em relação ao foco de atenção dado pelos estudiosos do clima organizacional, pode ser traçado um paralelo entre a literatura nacional e a internacional. Os pesquisadores americanos, por exemplo, começaram a estudar essa temática na década de 1960, época na qual foi publicado um dos primeiros artigos que discutiu a natureza dos fenômenos (Forehand \& Gilmer, 1964). No Brasil, os estudos dessa área tiveram início na década de 1970, com o trabalho de Saldanha intitulado Atmosfera Organizacional, em que o autor fazia um alerta sobre a importância do bem-estar psíquico dos indivíduos nas organizações. As pesquisas realizadas no Brasil interessavam-se pela investigação do efeito do clima entre outras variáveis do cenário organizacional, enquanto nos Estados Unidos, o foco eram as discussões sobre a própria natureza e origem do fenômeno.

De acordo com Puente-Palacios (2002), existem divergências na estrutura atribuída ao conceito do clima organizacional, e essas diferenças constituem um indicador da existência de correntes teóricas diversas. Os estudos de James e Jones (1974), Schneider (1975), Schneider e Reichers (1983), Koys e DeCotiis (1991), Moran e Volkwein (1992) e Puente-Palacios (2002) demonstram que o clima é composto por variáveis que interagem entre si.

A partir dos estudos de clima fica evidente que os autores da área não apresentam consenso nas dimensões constitutivas deste fenômeno, como exemplificado pelos estudos de Martins, Oliveira, Silva, Pereira e Souza (2004) e Puente-Palacios (2002). De acordo com Puente-Palacios e Carneiro (2005), a dificuldade em delimitar as dimensões constitutivas de clima é o desafio que circula as pesquisas da área. Os autores concordam que há necessidade de realizar pesquisas com o objetivo de definir de maneira clara os limites do conceito nos quais se pretende trabalhar.

A partir dessas verificações, fica evidente que é preciso fazer uma revisão rígida dos elementos essenciais do construto, pois muito daqueles assinalados como componentes são, de fato, construtos teoricamente diferentes do clima organizacional. Por essa razão, Puente-Palacios (2002) afirma ser incoerente incluí-los como dimensões a serem investigadas por uma escala de avaliação do clima organizacional. Essa autora sugere, portanto, que os pesquisadores interessados nesse campo restrinjam os elementos considerados constitutivos do clima e sintetizem os tópicos a serem investigados. Essa sugestão caminha para a redefinição do construto e, na adoção da denominação definitiva de clima social, abreviando sua abrangência àquilo que concede ao construto natureza específica, permite distingui-lo dos outros conceitos que permeiam o campo das organizações.

Nesse cenário, surgiram dois autores que realizaram um importante estudo sobre o campo teórico do clima: Koys e DeCotiis. Em 1991, eles avaliaram todos os estudos dos autores da área com o objetivo de identificar as dimensões propostas como constituintes de clima. Encontraram 80 dimensões e as caracterizaram de forma a agrupar e eliminar aquelas que fazem parte de outros constructos. Os resultados encontrados apontaram para oito dimensões constitutivas de clima: autonomia, coesão, confiança, pressão, suporte, reconhecimento, igualdade e inovação. A partir desse estudo, Koys e DeCotiis (1991) concluíram, ainda, que o clima é um fenômeno de descrição multidimencional a partir das percepções dos empregados e de suas experiências dentro de uma organização. Segundo os autores, baseado no acúmulo de experiência dentro de uma organização, as pessoas obtêm percepções. Essas percepções servem como um mapa cognitivo individual de como funciona a organização e, portanto, ajudam a determinar qual é o comportamento apropriado em cada 
situação. Assim, o clima é útil para adaptar o comportamento individual às demandas da vida na organização.

Segundo Koys e DeCotiis (1991), as percepções de clima têm quatro características: (a) o clima resume uma descrição individual da experiência organizacional, ao invés de reações afetivas ou avaliativas as quais têm sido experimentadas (embora as descrições não possam ser completamente separadas de suas evoluções, as distinções entre reações descritivas e avaliativas das experiências organizacionais distinguem clima de satisfação no trabalho); (b) as percepções do clima são relativamente estáveis; (c) são elas amplamente compartilhadas pelos membros da organização; (d) múltiplos climas podem existir dentro da mesma organização, desde que esta esteja presente em diferentes locais.

A partir dessas considerações, Koys e DeCotiis (1991, p. 266) definem o clima como um fenômeno multidimensional, percebido e compartilhado pelos membros de uma mesma organização, o qual influencia o comportamento individual. Adicionalmente, Puente-Palacios (2002) menciona que o clima não é uma característica estática, mas dinâmica, do âmbito organizacional, por isso a sua estrutura não está apenas definida pela presença-ausência de determinados elementos, mas também pelo tipo de relação estabelecida entre eles.

Assim como concluíram Koys e DeCotiis (1991) a respeito do clima organizacional, PuentePalacios e Freitas (2006) apontam que as dimensões que compõem o clima social devem ser reduzidas ao núcleo central do construto, de forma a não invadir campos teóricos conceitualmente diferenciados, como o da satisfação e o da veiculação afetiva. Nesse sentido, ao adaptar uma escala de clima social ao contexto de equipes de trabalho, Puente-Palacios e Carneiro (2005) afirmaram que as investigações relativas ao clima social devem incluir autonomia, inovação, gerenciamento das relações entre gestor e colaborador, valorização, e reconhecimento do desempenho.

Com as dimensões apontadas por Koys e DeCotiis (1991), a partir das argumentações teóricas e definições empíricas dos limites desse conceito, é possível concluir que o clima se refere a percepções construídas e sustentadas socialmente a respeito da organização.

O clima social, portanto, é definido e adotado neste estudo como um fenômeno multidimensional, relativamente estável, formado pela percepção compartilhada dos membros da organização sobre as experiências no trabalho, e tem a função de influenciar o comportamento individual.

Ao revisar o efeito do clima no contexto das organizações, verifica-se que ele tem sido amplamente apontado como fenômeno que produz efeitos na satisfação das pessoas. Nesse sentido, o presente estudo torna-se pertinente, uma vez que busca identificar o impacto do clima social no bemestar do trabalhador. Este trabalho, ainda, quer compreender o impacto de coping no bem-estar das pessoas nas organizações. A seguir serão apresentados o construto de coping, definições e conceito adotados neste estudo.

\section{Coping}

De acordo com Folkman e Moskowitz (2004), há mais de quatro décadas surgiram diversas pesquisas sobre coping em ciências sociais, comportamentais, medicina, saúde pública e enfermagem. Segundo estes autores, as pesquisas desta área buscam entender porque os indivíduos têm maneiras diferentes de lidar com o estresse em suas vidas.

Segundo Folkman e Moskowitz (2004), apesar de a maioria das pesquisas de coping ser baseada na psicologia do ego e em mecanismos de defesa, em 1966, em seu livro chamado Psychological Stress and the Coping Process, Richard Lazarus's apresentou uma explicação contextual ao estresse, a partir do estudo de coping. Assim, ele tirou o foco da patologia e apresentou uma gama de estratégias 
cognitivas e respostas comportamentais que as pessoas utilizam para se livrar do estresse e dos problemas da vida diária. Com este estudo, o campo ganhou raiz, estendeu-se pelas duas décadas seguintes, e várias pesquisas apontaram para uma nova perspectiva com relação a coping.

De acordo com Suls, David e Harvey (1996), essa nova tendência buscou enfatizar os comportamentos de coping e seus determinantes cognitivos e situacionais. Segundo Folkman e Lazarus (1985), pesquisadores passaram a conceituar coping como um processo transacional entre a pessoa e o ambiente, com ênfase tanto no processo, quanto em traços de personalidade. Lazarus e Folkman (1984) definiram coping através dos comportamentos que as pessoas usam para equilibrar as exigências internas e externas frente a situações estressantes. Segundo Folkman e Moskowitz (2004), esta definição foi amplamente aceita e, a partir dela, surgiram as principais publicações da área.

Retomando Lazarus e Folkman (1984), nessa perspectiva, considera-se que a resposta de enfrentamento é uma ação intencional, física ou mental, iniciada em resposta a um estressor percebido, dirigida para circunstâncias externas ou estados internos. Define-se, neste estudo, coping, portanto, como uma variável individual representada pelas formas como as pessoas comumente reagem ao estresse, determinadas por fatores pessoais, exigências situacionais e recursos disponíveis.

Segundo Antoniazzi, Dell'Aglio e Bandeira (1998), por sua vez, a resposta de estresse é qualquer ação envolvendo uma reação emocional ou comportamental espontânea. Ainda de acordo com estes autores, o objetivo do enfrentamento consiste na intenção de uma resposta, geralmente orientada para a redução do estresse. Ao propor esse esquema, os autores salientam a diferença entre resultados de coping, que são as consequências específicas da resposta de enfrentamento, e os resultados de estresse, ou seja, as consequências imediatas da resposta de estresse. E, segundo eles, ambos podem promover, ou não, o bem-estar.

Folkman e Lazarus (1980) salientam que a ação de enfrentamento pode ser direcionada internamente ou externamente. Quando o enfrentamento focalizado no problema é dirigido para uma fonte externa de estresse, inclui estratégias tais como negociar para resolver um conflito interpessoal ou solicitar ajuda prática de outras pessoas. O enfrentamento focalizado no problema e dirigido internamente, geralmente inclui reestruturação cognitiva como, por exemplo, a redefinição do elemento estressor.

Embora os estilos possam influenciar a extensão das estratégias de coping selecionadas, eles são fenômenos distintos, e têm diferentes origens teóricas. Esta distinção se faz necessária, uma vez que, neste estudo, serão pesquisadas as estratégias de enfrentamento. Especificamente serão estudadas as ações do sujeito que podem impactar no bem-estar no trabalho: (a) manejo, (b) controle ou (c) esquiva.

Segundo Pinheiro (2002), apesar da grande variedade de estudos conduzidos sobre coping nas últimas décadas, poucos trabalhos têm sido desenvolvidos para verificar a utilização de estratégias de coping voltadas para o ambiente ocupacional, justificando, assim, a inclusão dessa variável neste estudo.

\section{Problema, Objetivos e Modelo de Pesquisa}

Autores como Ryff e Keyes (1995), Ryan e Deci (2001), Nix, Ryan, Manly e Deci (1999) e Paz (2004) discutiram a importância do estudo do bem-estar no ambiente organizacional, porém, não apresentaram pesquisas que relacionassem esse bem-estar com clima social. Os estudiosos de clima social (Billings \& Moos, 1991; Martins, Oliveira, Silva, Pereira, \& Souza, 2004; Puente-Palacios \& Carneiro, 2005; Puente-Palacios \& Freitas, 2006; Schneider \& Reichers, 1983) também não apontaram ligação entre tais temas. Há estudos empíricos (Koys \& DeCotiis, 1991; Paz, 2004; Puente-Palacios, 2002; Warr, 1994, 2006), no entanto, que apontam para possibilidades de impacto entre essas variáveis, tais como relações interpessoais, dimensão social do trabalho, reconhecimento, suporte 
ambiental. Dessa forma, é possível indagar se realmente há relação entre o bem-estar ocupacional e o clima social.

Sobre a relação das variáveis: idade, gênero, estado civil e escolaridade com o bem-estar no trabalho, pode-se destacar a escassez de estudos empíricos sobre o efeito preditor de variáveis demográficas. Já no campo do bem-estar geral, variáveis como idade, gênero e estado civil já foram amplamente estudadas como preditoras. Diener, Suh, Lucas e Smith (1999) apresentou uma revisão sobre as variáveis relacionadas com as três dimensões do bem-estar subjetivo. Segundo o autor, inúmeros pesquisadores acreditavam, inicialmente, que as variáveis demográficas tivessem forte impacto sobre o bem-estar, no entanto, perceberam que o efeito dessas variáveis é pequeno e, quando encontrado, depende de fatores de personalidade.

Ainda cabe considerar que a percepção do bem-estar pode ser impactada por coping. Pois, segundo Antoniazzi et al. (1998), a resposta de estresse é qualquer ação envolvendo uma reação emocional ou comportamental espontânea que pode impactar o bem-estar. A partir dessa constatação, além de indagar se o clima social tem impacto sobre a percepção do bem-estar organizacional, cabe ainda questionar se coping também impacta na percepção do bem-estar.

Assim, o problema desta pesquisa é: qual o impacto dos dados demográficos (gênero, estado civil, idade e escolaridade), do clima social e do coping sobre o nível de bem-estar no trabalho?

O objetivo geral da pesquisa é identificar a relação entre clima social, coping e variáveis demográficas (gênero, estado civil, idade e escolaridade) no bem-estar de indivíduos no trabalho, conforme modelo apresentado na Figura 1.

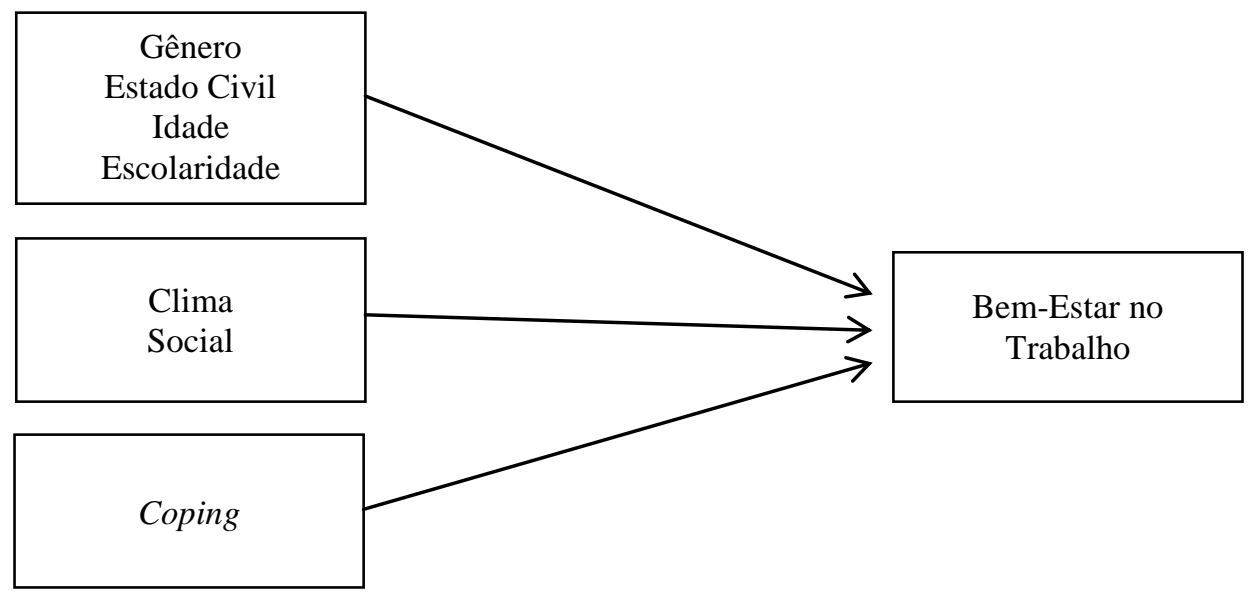

Figura 1. Modelo da Pesquisa.

\section{Método}

Este estudo caracterizou-se como correlacional (Sekaran, 1992), cujo interesse foi relacionar as variáveis gênero, estado civil, idade, escolaridade, clima social e estratégias de enfrentamento e bemestar no trabalho. A unidade de análise é individual, pois o foco é no impacto da percepção individual sobre o bem-estar. A pesquisa, por sua vez, é de corte transversal, uma vez que os dados foram coletados de uma só vez em um período de 15 dias. Já as escalas utilizadas nesta pesquisa foram aplicadas durante o programa de melhoria do clima organizacional, pela área de Recursos Humanos, em colaboradores de uma organização filantrópica confessional do ramo de educação privada, com 4.400 colaboradores. Essa organização atua em 16 estados do Brasil, divididos em 101 unidades: 21 colégios, 2 faculdades, 46 unidades sociais, 6 centros pastorais, 8 centros de formação e eventos, 9 
teatros, 2 museus, 1 radio web, 4 centros de produção de TV e vídeo, 1 casa de espetáculo multifuncional, e 1 Escritório Central (sede).

Do universo, compôs-se a amostra: 2.560 colaboradores concordaram em responder o questionário, totalizando $58 \%$ da população. A organização disponibilizou, para a realização deste estudo, o banco de dados da pesquisa e, ainda, documentos oficiais como livros, que descrevem a história; cartas, que descrevem a personalidade do fundador; o planejamento estratégico, política de cargos, carreiras e benefícios. Foi feita, ainda, a análise do site oficial da organização.

\section{Instrumentos de coleta dos dados}

Os instrumentos de coleta de dados utilizados nessa pesquisa foram: as escalas de bem-estar no trabalho (EBET), de Paschoal e Tamayo (2008); a avaliação de clima social (EACS), elaborada por Puente-Palacios e Carneiro (2005), adaptada para o contexto organizacional; e coping ecupacional (ECO), desenvolvida por Latack (1986) e traduzida e adaptada para a língua portuguesa por Pinheiro, Tróccoli e Tamayo (2003).

A escala de bem-estar no trabalho (EBET) foi desenvolvida e validada por Paschoal e Tamayo (2008), e é composta por três fatores: afeto positivo, com nove itens (ex.: Animado); afeto negativo, com 12 itens (ex.: Nervoso); realização, com nove itens (ex.: Realizo atividades que expressam minhas capacidades), totalizando 31 itens. Para a amostra desta pesquisa, obtiveram-se os seguintes índices de confiabilidade (alpha de Cronbach): afeto positivo $\alpha=0,93$; afeto negativo $\alpha=0,91$; e realização $\alpha=0,88$.

A escala elaborada por Puente-Palacios e Carneiro (2005) - EACS foi validada no contexto de equipes de trabalho. Essa escala foi adaptada ao contexto organizacional para este estudo, trocando-se equipes por organização. Ela é composta por 35 itens respondidos em escala Likert de 5 pontos (1=Nunca; $2=$ Poucas vezes; $3=$ Às vezes; $4=$ Quase sempre; $5=$ Sempre). A análise fatorial apontou para a presença de três fatores. Para a rotação e a extração dos fatores, foi utilizado o método de fatoração dos eixos principais com rotação oblíqua. A estrutura encontrada com três fatores apresentou sentido teórico: o primeiro ficou constituído pelos itens de inovação e desempenho $(\alpha=0,95)$; o segundo, pelos itens de reconhecimento $(\alpha=0,94)$; e o terceiro constituído pelos itens de autonomia $(\alpha=0,84)$. Após o tratamento estatístico, os itens que compunham a faceta relação gestor x colaborador na versão original foram distribuídos entre os três fatores. O item 26 (os gestores são compreensivos com os colaboradores) foi excluído por apresentar carga fatorial acima de 0,30 em dois fatores.

A escala de coping ocupacional - ECO, descrita neste estudo como estratégias de enfrentamento ao estresse, foi desenvolvida por Latack (1986), traduzida e adaptada para a língua portuguesa por Pinheiro et al. (2003). Os índices de confiabilidade para a presente amostra foram: Controle $(\alpha=$ $0,78)$, Esquiva $(\alpha=0,77)$ e Manejo de Sintomas $(\alpha=0,81)$.

\section{Resultados}

Após verificação dos pressupostos para as análises, foram realizadas correlações entre todos os fatores pesquisados, conforme Tabela 1 . O resultado demonstrou que os fatores de clima são altamente correlacionados entre si, provocando, assim, efeito de supressão na regressão. Dentre esses, autonomia apresentou correlação positiva e de moderada a alta com bem-estar, mas os fatores inovação, desempenho e reconhecimento apresentaram correlações mais fortes. No caso dos fatores de coping, esquiva teve resultado fraco na correlação, porém manejo e controle tiveram resultado significativo. 


\section{Tabela 1}

\section{Média, Desvio-padrão e Correlações entre as Variáveis do Estudo}

\begin{tabular}{|c|c|c|c|c|c|c|c|c|c|c|c|c|}
\hline \multirow[t]{2}{*}{ Fatores } & \multirow[t]{2}{*}{ Média } & \multirow{2}{*}{$\begin{array}{l}\text { Desvio- } \\
\text { padrão }\end{array}$} & \multicolumn{10}{|c|}{ Correlações } \\
\hline & & & Idade & $\begin{array}{l}\text { Afeto } \\
\text { positivo }\end{array}$ & $\begin{array}{l}\text { Afeto } \\
\text { negativo }\end{array}$ & Realização & Manejo & Controle & Esquiva & $\begin{array}{l}\text { Inov e } \\
\text { desemp }\end{array}$ & $\begin{array}{l}\text { Reconhe- } \\
\text { cimento }\end{array}$ & $\begin{array}{l}\text { Auto- } \\
\text { nomia }\end{array}$ \\
\hline Idade & 26,59 & 18,69 & 1 & & & & & & & & & \\
\hline A positivo & 3,43 & 0,84 & $0,168 * *$ & 1 & & & & & & & & \\
\hline A negativo & 1,82 & 0,67 & $-0,146^{* *}$ & $-0,588^{* *}$ & 1 & & & & & & & \\
\hline Realização & 4,07 & 0,62 & $0,147 * *$ & $0,610 * *$ & $-0,400 * *$ & 1 & & & & & & \\
\hline Manejo & 2,86 & 0,68 & 0,028 & $0,257 * *$ & $-0,173 * *$ & $0,221 * *$ & 1 & & & & & \\
\hline Controle & 3,38 & 0,53 & 0,011 & $0,214 * *$ & $-0,061 * *$ & $0,255^{* *}$ & $0,369 * *$ & 1 & & & & \\
\hline Esquiva & 2,82 & 0,68 & $-0,057 * *$ & 0 & $-0,044^{*}$ & 0,013 & $0,415^{* *}$ & $0,348 * *$ & 1 & & & \\
\hline Inov. e des. & 3,67 & 0,91 & $0,183^{* *}$ & $0,610 * *$ & $-0,495^{* *}$ & $0,552 * *$ & $0,250 * *$ & $0,246 * *$ & $-0,009$ & 1 & & \\
\hline Reconheci. & 3,62 & 1,06 & $0,189 * *$ & $0,637 * *$ & $-0,515^{* *}$ & $0,533^{* *}$ & $0,221 * *$ & $0,185^{* *}$ & $-0,028$ & $0,859 * *$ & 1 & \\
\hline Autonomia & 3,68 & 0,88 & $0,160 * *$ & $0,520 * *$ & $-0,408 * *$ & $0,462 * *$ & $0,222 * *$ & $0,232 * *$ & 0,009 & $0,787 * *$ & $0,726^{* *}$ & 1 \\
\hline
\end{tabular}


A seguir, foram realizadas as regressões stepwise em blocos para cada fator de bem-estar. A Tabela 2 apresenta o modelo final das regressões para os três fatores de bem-estar. Para afeto positivo, pode-se observar que a variável demográfica idade apresentou contribuição significativa, assim como coping (manejo, controle, esquiva) e clima social (reconhecimento, inovação e desempenho). Para afeto negativo, idade, escolaridade, coping e clima social apresentaram contribuição significativa. Para realização, escolaridade, idade e gênero, além do coping e do clima social, apresentaram contribuição significativa, sendo que os modelos explicaram entre 31 a $43 \%$ da variância de bem-estar.

Tabela 2

Resultados da Regressão para Afeto Positivo

\begin{tabular}{lllllll}
\hline & \multicolumn{2}{l}{ Afeto positivo } & \multicolumn{2}{l}{ Afeto negativo } & Realização & \\
\cline { 2 - 6 } Variáveis & $\boldsymbol{\beta}$ & $\mathbf{R}\left(\mathbf{R}^{2}\right)$ & $\boldsymbol{\beta}$ & $\mathbf{R}\left(\mathbf{R}^{2}\right)$ & $\boldsymbol{\beta}$ & $\mathbf{R}\left(\mathbf{R}^{2}\right)$ \\
\hline Idade & 0,017 & & $-0,040^{*}$ & & $0,061^{* *}$ & \\
Escolaridade & ---- & & $0,138^{* *}$ & & $0,055^{*}$ & \\
Gênero & --- & & --- & & $0,043^{*}$ & \\
Manejo & $0,099^{* *}$ & $0,659^{* *}$ & $-0,047^{*}$ & $0,557^{* *}$ & $0,062^{* *}$ & $0,563^{* *}$ \\
Controle & $0,057^{* *}$ & $(0,434)$ & ---- & $(0,310)$ & $0,133^{* *}$ & $(0,316)$ \\
Esquiva & $-0,027$ & & $-0,001$ & & $-0,031$ & \\
Reconheci-mento & $0,405^{* *}$ & & $-0,353^{* *}$ & & $0,232^{* *}$ & \\
Inovação e & $0,223^{* *}$ & & $-0,197^{* *}$ & & $0,256^{* *}$ & \\
desempenho & & & & & & \\
\hline
\end{tabular}

Nota. ${ }^{*} \mathrm{p}<0,05,{ }^{*} \mathrm{p}<0,01,----$ variável ausente no modelo.

Ressalta-se que, em função do efeito de supressão, o fator de clima autonomia não foi considerado nas regressões múltiplas. Entretanto, isso não quer dizer que esse fator não seja importante para o bem-estar no trabalho, pois, na tabela de correlação, pode-se ver que autonomia apresenta correlação positiva de moderada a alta com bem-estar.

\section{Discussão}

No presente estudo, foram testadas as variáveis idade, gênero, estado civil e escolaridade. Os resultados das correlações apontaram relações significativas entre eles com pelo menos um dos fatores de bem-estar no trabalho, mas as relações foram fracas, em conformidade com o que afirma Diener $e t$ al. (1999). Segundo o autor, inúmeros pesquisadores acreditavam, inicialmente, que as variáveis demográficas tivessem forte impacto sobre o bem-estar, no entanto perceberam que o efeito dessas variáveis é pequeno e, quando encontrado, depende de fatores de personalidade.

Destaca-se a idade, que contribuiu na variância de bem-estar no trabalho. Pesquisas no campo do bem-estar geral sugerem que a satisfação com a vida tende a aumentar com a idade ou, pelo menos, não tende a diminuir (Horley \& Lavery, 1995). Enquanto proporção de anos, na organização estudada, é possível associar idade ao tempo de casa ao considerar que esta impacta indiretamente no plano de carreira, uma vez que são oferecidos ganhos a partir do acúmulo de anos no contrato de trabalho (tempo de casa, como já dito). Assim pode haver um impacto indireto dessas medidas sobre o nível de bem-estar no trabalho.

No campo de bem-estar geral, parece não haver dados consistentes que suportem a diferença de 
bem-estar entre homens e mulheres (Diener, Suh, Lucas, \& Smith, 1999). Neste estudo, na regressão, gênero não impactou na explicação da variância de afetos e teve pouca influência sobre realização. Esses resultados são consistentes com as pesquisas sobre bem-estar geral.

Também nos estudos de bem-estar geral, pesquisas demonstraram que pessoas casadas tendem a apresentar bem-estar maior do que pessoas divorciadas, separadas, viúvas ou solteiras. Entre os adultos que não são casados, aqueles que vivem com um parceiro apresentam maior bem-estar. Isso porque a presença de um parceiro pode significar maior oportunidade de suporte social (Diener et al., 1999). Neste estudo, estado civil não apresentou relação significativa na variância de bem-estar no trabalho, tanto nos resultados das correlações quanto nas regressões.

Sobre o impacto da escolaridade sobre o bem-estar, não há estudos empíricos que expliquem essa relação, mesmo no campo do bem-estar geral, embora se saiba que o nível de escolaridade esteja ligado diretamente a aumento de renda. Neste estudo, escolaridade impactou positivamente sobre a variância do bem-estar no trabalho. Tais resultados podem ser explicados uma vez que a organização pesquisada é do ramo de educação e, em seu quadro de colaboradores, a maioria são professores, que são reconhecidos em plano de careira a partir do indicador de acúmulo de títulos. Ainda a própria organização oferece cursos de pós-graduação para seus professores. Cabe ressaltar que colaboradores técnicos, administrativos e docentes são aproveitados em processos de seleção internos quando surgem vagas, gerando promoções e sentimento de estabilidade. Nesse caso, escolaridade é um fator que permeia a avaliação.

Sobre a relação entre o clima social e o bem-estar no trabalho, nos estudos sobre o bem-estar geral, o relacionamento interpessoal positivo e a autonomia aparecem como fortes preditores do bemestar no trabalho. No modelo de Ryff e Keyes (1995), o relacionamento interpessoal é uma dimensão do bem-estar psicológico, além da autonomia. Ryan e Deci (2001) apresentam a autonomia e os relacionamentos interpessoais positivos como duas das três necessidades psicológicas básicas que, quando satisfeitas, levam ao bem-estar no trabalho; a outra é a competência. $O$ resultado das correlações demonstrou relação significativa entre autonomia e bem-estar no trabalho, dando apoio ao que afirmam Ryan e Deci (2001).

No presente estudo, a escala de clima social apresentou itens que exploraram a qualidade das relações interpessoais, de autonomia, inovação, desempenho e reconhecimento sobre os fatores de bem-estar no trabalho. Importante ressaltar que, apesar da construção dos fatores após validação não apresentar o termo relações interpessoais, a escala contempla nove itens que exploram a relação entre gestor e colaborador. Sendo assim, os resultados das correlações e regressões dão apoio à hipótese de que as relações interpessoais são importantes para o bem-estar.

Segundo Puente-Palacios e Carneiro (2005), pesquisas que busquem entender os efeitos do clima no ambiente organizacional podem contribuir para a melhoria da qualidade de vida do homem no trabalho. Os resultados das correlações e regressões apontaram para esse objetivo. As correlações indicaram relação significativa entre clima e bem-estar no trabalho, positiva entre os fatores de clima, afeto positivo e realização, e negativa com afeto negativo. Na regressão, inovação, desempenho e reconhecimento contribuíram significativamente para a explicação da variância de bem-estar no trabalho, com destaque para o reconhecimento, que acrescentou $31 \%$ de explicação do afeto positivo e $24 \%$ do afeto negativo. Ainda que brevemente apontado na seção de resultados deste trabalho, vale destacar novamente que ocorreu efeito de supressão do fator autonomia, fazendo com que ele não aparecesse nos resultados das regressões.

Em relação ao impacto de coping sobre o bem-estar no trabalho, concluiu-se, tanto a partir das análises de correlação quanto de regressão que dois dos três fatores (manejo, controle e esquiva) estão correlacionados significativamente e impactam na variância do bem-estar no trabalho. São eles: manejo e controle, com destaque para manejo que contribuiu com $6 \%$ na variância.

As regressões apontaram que coping impacta no bem-estar no trabalho e vai ao encontro da definição de Lazarus e Folkman (1984), adotada nesse estudo de que tal processo pode ser entendido 
como uma variável individual representada pelas formas como as pessoas comumente reagem ao estresse, determinadas por fatores pessoais, exigências situacionais e recursos disponíveis, que favorece o bem-estar dos indivíduos.

\section{Conclusão}

A partir dos resultados dessa pesquisa, conclui-se que as variáveis demográficas, o clima social e o coping impactam no bem-estar no trabalho. Todavia, as variáveis demográficas - gênero, idade, estado civil e escolaridade - apontaram relações fracas. Assim, é possível afirmar que o contexto de trabalho é mais importante para favorecer o nível de bem-estar dos trabalhadores do que características demográficas. Além da ênfase no contexto, habilidades pessoais para lidar com as demandas do ambiente de trabalho também contribuem para proporcionar bem-estar. O objetivo geral foi alcançado e os resultados encontrados nessa pesquisa corroboram a ideia de Puente-Palacios (2002), de que é fundamental a importância outorgada ao papel do líder e dos pares na atribuição de significado à dinâmica organizacional. Esse dado leva a ressaltar a possibilidade de abordar o fenômeno sob a denominação de clima social da organização.

Os resultados encontrados respondem ao objetivo que buscava saber se clima é antecedente de bem-estar, e os resultados demonstraram que o aspecto não só se relaciona como impacta fortemente no bem-estar no trabalho, corroborando com a literatura da área. O clima apresentou-se como o mais forte preditor de bem-estar no trabalho.

Sobre coping, apesar da grande variedade de estudos conduzidos nas últimas décadas, poucos trabalhos têm sido desenvolvidos para verificar a utilização dessas estratégias voltadas para o ambiente de trabalho, portanto, os pesquisadores da área podem considerar os resultados encontrados nesse estudo como a abertura de um campo ainda não explorado. Outras pesquisas que analisem o impacto de coping no bem-estar no trabalho podem contribuir para o entendimento da dinâmica deste, principalmente, em tempos em que o aumento da competitividade no mundo dos negócios, das novas demandas do ambiente de trabalho, tempos de globalização e mudanças de mercado marcam o aumento significativo das demandas no ambiente profissional, especialmente, com a epidemia de burnout.

Ainda que o estudo tenha alcançado os objetivos propostos e tenha oferecido contribuições ao campo do bem-estar no trabalho, há limitações que merecem ser destacadas, pois apontam para questões que devem ser melhor desenvolvidas em pesquisas futuras. Dentre essas limitações, as principais foram: a área de recursos humanos da própria organização coletou os dados e, apesar dos cuidados com a preservação do anonimato dos participantes, pode ter havido tendenciosidade nas respostas por medo de retaliações ou receio de identificação dos respondentes; outra limitação que pode ser destacada consiste na dificuldade de generalização dos resultados encontrados. A pesquisa foi conduzida em uma única organização, portanto, fatores relacionados a cultura, valores e tradições podem ter atravessado interpretações dos itens dos instrumentos. Por outro lado, a diversidade das unidades que compõem a organização favorece a possibilidade de generalização. A terceira limitação diz respeito ao tipo da organização pesquisada, que é confessional, filantrópica, do ramo de educação. Essas características especificam o foco do objetivo institucional e pré-determinam alguns comportamentos no ambiente de trabalho.

Ainda que as dimensões aqui adotadas tenham contribuído para a compreensão do bem-estar no trabalho, pesquisas vindouras podem suprir essas limitações sendo conduzidas em outras cidades e regiões do país, em diversos ramos do mercado de trabalho, verificando diferenças entre organizações públicas e privadas de diferentes ramos de atuação. Além disso, o conteúdo das dimensões utilizadas deve ser aprofundado.

\section{Artigo recebido em 18.05.2011. Aprovado em 04.01.2012.}




\section{Referências}

Albuquerque, A. S., \& Trócolli, B. T. (2004). Desenvolvimento de uma escala de bem-estar subjetivo. Psicologia: Teoria e Pesquisa, 20(2), 153-164. doi: 10.1590/S0102-37722004000200008.

Antoniazzi, A. S., Dell'Aglio, D. D., \& Bandeira, D. R. (1998). O conceito de coping. Estudos de Psicologia, 3(2), 273-294. doi: 10.1590/S1413-294X1998000200006

Billings, A. G., \& Moos, R. H. (1991). Undestanding and improving climates. In J. W. Jones, B. D. Steffy, \& D. W. Bray (Eds.), Applying psychology in business: the handbook for managers and human resource professionals (pp. 552-562). Lexington, MA: D.C. Health.

Brief, A. P., \& Roberson, L. (1989). Job attitude organization: an exploratory study. Journal of Applied Social Psychology, 19(9), 717-727. doi: 10.1111/j.1559-1816.1989.tb01254.x

Brief, A. P., \& Weiss, H. M. (2002). Organizational behavior: affect in the workplace. Annual Review of Psychology, 53, 279-307. doi: 10.1146/annurev.psych.53.100901.135156

Brooks, B. A., \& Anderson, M. A. (2005). Defining quality of nursing work life. Nursing Economics, 23(6), 319-326.

Daniels, K. (2000). Measures of five aspects of affective well-being at work. Human Relations, 53(2), 275-294.

Dessen, M. C., \& Paz, M. G. T. (2010a). Bem-estar pessoal nas organizações: o impacto de configurações de poder e características de personalidade. Psicologia: Teoria e Pesquisa, 26(3), 549-556. doi: 10.1590/S0102-37722010000300018

Diener, E. (1984). Subjective well-being. Psychological Bulletin, 95(3), 542-575. doi: 10.1037/00332909.95.3.542

Diener, E., Suh, E. M., Lucas, R. E., \& Smith, H. L. (1999). Subjective well-being: three decades of progress. Psychological Bulletin, 125(2), 276-302. doi: 10.1037//0033-2909.125.2.276

Doest, L. ter, Maes, S., Gebhardt, W. A., \& Koelewijn, H. (2006). Personal goal facilitation through work: implications for employee satisfaction and well-being. Applied Psychology, 55(2), 192219. doi: $10.1111 / \mathrm{j} .1464-0597.2006 .00232 . x$

Folkman, S., \& Lazarus, R. S. (1980). An analysis of coping in a middle-aged community sample. Journal of Health and Social Behavior, 25(3), 219-239.

Folkman, S., \& Lazarus, R. S. (1985). If it changes it must be a process: a study of emotion and coping during three stages of a college examination. Journal of Personality and Social Psychology, $48(1), 150-170$.

Folkman, S., \& Moskowitz, J. T. (2004). Coping: pitfalls and promise. Annual Reviews Psychology, 55(1), 745-774. doi: 10.1146/annurev.psych.55.090902.141456

Forehand, S., \& Gilmer, H. (1964). Environmental variation an studies of organizacional behavior. Psychological Bulletin, 62(6), 361-383. doi: 10.1037/h0045960

Horley, J., \& Lavery, J. J. (1995). Subjective well-being and age. Social Indicators Researchm, 34(2), 275-282. doi: 10.1007/BF01079200

Horn, J. E. van, Taris, T. W., Schaufel, W. B., \& Schreurs, P. J. G. (2004). The structure of occupational well-being: a study among Dutch teachers. Journal of Occupational and Organizational Psychology, 77(3), 365-375. doi: 10.1348/0963179041752718 
House, J. S. (1981). Work stress and social support. Massachusetts: Addison-Wesley Publishing Company.

James, L. R., \& Jones, A. (1974). Organizational climate: a review of theory and research. Psychological Bulletin, 81(12), 1096-1112. doi: 10.1037/h0037511

Koys, D., \& DeCotiis, T. (1991). Inductive measures of psychological climate. Human Relations, 44(3), 265-285. doi: 10.1177/001872679104400304

Latack, J. C. (1986). Coping with job stress: measures and future directions for scale development. Journal of Applied Psychology, 71(3), 377-385. doi: 10.1037/0021-9010.71.3.377

Lazarus, R. S., \& Folfman, S. (1984). Coping and adaptation. In W. D. Gentry (Ed.), Handbook of Behavioral Medicine (pp. 282-325). New York: The Guilford Press.

Martins, M. C. F. do, Oliveira, B. de, Silva, C. F. da, Pereira, K. C., \& Souza, M. R. de (2004). Construção e validação de uma escala de medida de clima organizacional. Revista Psicologia: Organização e Trabalho, 4(1), 37-60.

Moran, E., \& Volkwein, J. (1992). The cultural approach to the formation of organizational climate. Human Relations, 45(1), 19-47. doi: 10.1177/001872679204500102

Nadler, D. A., \& Lawler, E. E. (1983). Quality of work life: perspectives and directions. Organizational Dynamics, 11(3), 20-30. doi: 10.1016/0090-2616(83)90003-7

Nix, G. A., Ryan, R. M., Manly, J. B., \& Deci, E. L. (1999). Revitalization through self-regulation: the effects of autonomous and controlled motivation on happiness and vitality. Journal of Experimental Social Psychology, 35(3), 266-284. doi: 10.1006/jesp.1999.1382

Paschoal, T. (2008). Bem-estar no trabalho: relações com suporte organizacional, prioridades axiológicas e oportunidades de alcance de valores pessoais no trabalho (Tese de doutorado). Universidade de Brasília, Brasília, DF, Brasil.

Paschoal, T., \& Tamayo, A. (2008). Construção e validação da escala de bem-estar no trabalho. Avaliação Psicológica, 7(1), 11-22.

Paschoal, T., Torres, C. V., \& Porto, J. B. (2010). Felicidade no trabalho: relações com suporte organizacional e suporte social. Revista de Administração Contemporânea, 14(6), 1054-1072. Recuperado em http://www.scielo.br/scielo.php?pid=S141565552010000700005\&script=sci_arttext. doi: 10.1590/S1415-65552010000700005

Paz, M. G. T. (2004). Poder e saúde organizacional. In A. Tamayo (Org.), Cultura e saúde nas organizações (pp. 127-154). Porto Alegre: Artmed.

Paz, M. G. T. (2005). Bem-estar pessoal nas organizações: construção e validação de um instrumento de medida. (Texto não publicado). Universidade Católica de Brasília, Brasília, DF.

Pinheiro, F. A. (2002). Aspectos psicossociais dos distúrbios osteomusculares relacionados ao trabalho - DORT/LER (Tese de doutorado). Universidade de Brasília, Brasília, DF, Brasil.

Pinheiro, F. A., Tróccoli, B. T., \& Tamayo, M. R. (2003). Mensuração de coping no ambiente ocupacional. Psicologia: Teoria e Pesquisa, 19(2), 153-158. doi: 10.1590/S010237722003000200007

Puente-Palacios, K. E. (2002). Abordagens teóricas e dimensões empíricas do conceito de clima organizacional. Revista de Administração da USP, 37(3), 96-104. 
Puente-Palacios, K. E., \& Carneiro, B. P. (2005). Adaptação de uma escala de avaliação do clima social ao contexto de equipes de trabalho. Revista Psicologia: Organizações e Trabalho, 5(1), 45-70.

Puente-Palacios, K. E., \& Freitas, I. A. de (2006). Clima organizacional: uma análise de sua definição e de seus componentes. Organizações e Sociedade, 13(38), 45-57.

Rodrigues, M. V. C. (1995). Qualidade de vida no trabalho (2a ed.). Rio de Janeiro: Vozes.

Ryan, R. M., \& Deci, E. R. (2001). On happiness and human potentials: a review of research on hedonic and eudaimonic well-being. Annual Review of Psychology, 52, 141-166. doi: 10.1146/annurev.psych.52.1.141

Ryff, C. D. (1989). Hapiness is everything, or is it? Explorations on the meaning of psychological well-being. Journal of Personality and Social Psychology, 57(6), 1069-1081. doi: 10.1037/0022-3514.57.6.1069

Ryff, C. D., \& Keyes, C. L. M. (1995). The structure of psychological well-being revisited. Journal of Personality and Social Psychology, 69(4), 719-727. doi: 10.1037/0022-3514.69.4.719

Schneider, B. (1975). Organizational climate: an essay. Personnel Psychology, 28(4), 447-479.

Schneider, B., \& Reichers, A. (1983). On the etiology of climates. Personnel Psychology, 36(1), 1939. doi: 10.1111/j.1744-6570.1983.tb00500.x

Sekaran, U. (1992). Research methods for business: a skill-building approach (2a ed.). Nova York: John Wiley \& Sons.

Siqueira, M. M., \& Padovam, V. A. R. (2008). Bases teóricas de bem-estar subjetivo, bem-estar psicológico e bem-estar no trabalho. Psicologia: Teoria e Pesquisa, 24(2), 201-209. doi: 10.1590/S0102-37722008000200010

Sousa-Poza, A., \& Sousa-Poza, A. A. (2000). Well-being at work: a cross-national analysis of the levels and determinants of job satisfaction. Journal of Socio-Economics, 29(6), 517-538. doi: $10.1016 /$ S1053-5357(00)00085-8

Suls, J., David, J. P., \& Harvey, J. H. (1996). Personality and coping: three generations of research. Journal of Personality, 64(4), 711-735. doi: 10.1111/j.1467-6494.1996.tb00942.x

Tamayo, A. (2004). Introdução. In A. Tamayo (Ed.), Cultura e saúde nas organizações (pp. 11-16). Porto Alegre: Artmed.

Trist, E. (1975). Planning the first steps toward quality of working life in a developing conty. In L. Daves \& A. B. Cherns (Eds.), The quality of work life (pp. 78-85). London: Free Press.

Walton, R. E. (1973). Quality of working life: what is? Sloan Management Review, 15(1), 11-21.

Warr, P. (1994). A conceptual framework for the study of work and mental health. Work and Stress, 8(2), 84-97. doi: 10.1080/02678379408259982

Warr, P. (2006). Differential activation of judgments in employee well-being. Journal of Occupational and Organizational Psychology, 79(2), 225-244. doi: 10.1348/096317905X52652

Waterman, A. S. (1993). Two conceptions of happiness: contrasts of personal expressiveness (eudaimonia) and hedonic enjoyment. Journal Peronality and Social Psychology, 64(4), 678691. doi: 10.1037/0022-3514.64.4.678

Weiss, H. M., \& Cropanzano, R. (1996). Affective events theory: a theoretical discussion of the structure, causes and consequences of affective experiences at work. In B. M. Staw \& L. L. 
Cummings (Orgs.), Research in organizational behavior: an annual series of analytical essays and critical reviews (pp. 1-74). Greenwich, CT: JAI.

Werther, W. B., Jr., \& Davis, K. J. (1983). Administração de pessoal e recursos humanos. São Paulo: McGraw do Brasil. 